\title{
P198: Antimicrobial treatment for urinary tract infection (UTI) among patients having total hip (THA) or total knee arthroplasty (TKA)
}

\author{
L Herwaldt $^{\text {* }}$ S Bailin², B Johannsson', N Noiseux ${ }^{3}$, A Haleem¹, S Johnson ${ }^{4}$ \\ From 2nd International Conference on Prevention and Infection Control (ICPIC 2013) \\ Geneva, Switzerland. 25-28 June 2013
}

\section{Introduction}

Patients (pts) having THA or TKA were screened preoperatively (preop) and postoperatively (postop) for UTI with urinalysis (UA) and microscopic exam (micro), regardless of symptoms (sx).

\section{Objectives}

To assess the association between UA and micro results and antimicrobial treatment $(\mathrm{Rx})$ for UTI in pts having THA or TKA.

\section{Methods}

We reviewed records of pts who had THA $(n=100)$ or TKA ( $n=100$ ) between $21 / 2 / 2011-29 / 6 / 2011$ and we interviewed 50 pts who had THA or TKA between 21/ $5 / 2012-17 / 7 / 2012$ to assess the association between sxs of UTI and antimicrobial Rx. We used logistic regression to identify variables associated with antimicrobial Rx for UTI.

\section{Results}

190/200 (95\%) pts had UAs, 91\% had micros and 0.5\% had urine cultures preop. 37 (18.5\%) pts received antimicrobials for UTI preop. Positive leukocyte esterase $(\mathrm{LE} ; \mathrm{p}<.0001)$ and white blood cell $(\mathrm{WBC})$ count $>5$ $(\mathrm{p}=.0098)$ were associated with antimicrobial Rx for UTI preop. 198 (99\%) pts had UAs, 98\% had micros, and $2.5 \%$ had urine cultures after Foley removal. $72(36 \%)$ pts received postop antimicrobials for UTI. Positive $(+)$ LE $(\mathrm{p}<.0001), \mathrm{WBC}$ count $>5(\mathrm{p}=.014)$ and older age $(\mathrm{p}=.014)$ were associated with antimicrobials for UTI postop. Rx for UTI was related to LE level $(\mathrm{p}<.0001)$.
43/72 (59.7\%) pts Rxed for UTIs postop did not meet criteria for UTI. 28/50 (56\%) pts interviewed had sxs consistent with UTI but pts with sxs were not Rxed more often than pts without sx. $3 / 250$ pts $(1.2 \%)$ had $C$ difficile infection (CDI). On the basis of the data, practice was changed. Urine cultures are obtained from pts $\mathrm{w} /$ + LE and + nitrite or with sx of UTI. Pts with + cultures are treated for UTI.

\section{Conclusion}

45.5\% pts received antibiotics preop or postop for UTI; most did not meet criteria for UTI. LE results determined whether pts were Rx for UTI. Antimicrobial use and CDI among pts having THA or TKA could be reduced if only pts with UTI sxs are screened or if all pts are screened but only pts with + LE, nitrite, and cultures are treated.

\section{Disclosure of interest}

None declared.

\section{Author details \\ ${ }^{1}$ Internal Medicine, U of lowa College of Medicine, lowa City, IA, USA. ${ }^{2}$ University of lowa College of Medicine, lowa City, IA, USA. ${ }^{3}$ Orthopaedics, U of lowa College of Medicine, lowa City, IA, USA. ${ }^{4}$ Pharmaceutical Care, U of lowa Hospitals and Clinics (UIHC), lowa City, IA, USA.}

Published: 20 June 2013

\section{doi:10.1186/2047-2994-2-S1-P198}

Cite this article as: Herwaldt et al:: P198: Antimicrobial treatment for urinary tract infection (UTI) among patients having total hip (THA) or total knee arthroplasty (TKA). Antimicrobial Resistance and Infection Control 2013 2(Suppl 1):P198.

${ }^{1}$ Internal Medicine, U of lowa College of Medicine, lowa City, IA, USA

Full list of author information is available at the end of the article 